\title{
UNCONVENTIONAL SUPERCONDUCTIVITY
} \section{Suppressing superconductivity by adding it} Using electric field gating, researchers demonstrate switching from a single to a multi-condensate superconducting state at an oxide-based interface, and show that this transition leads to an overall weakening of the superconductivity.

\section{Hans Hilgenkamp \& Sander Smink}

One takes a superconducting material and adds more superconducting charge to it. The end result is a weaker superconductor than the original one. It sounds almost homeopathic but, writing in Nature Materials, this is exactly what Gyanendra Singh and colleagues have observed in their experiments on two-dimensional superconducting states residing at interfaces in oxide-based heterosystems [1].

The key material in their studies is strontium titanate $\left(\mathrm{SrTiO}_{3}\right)$, an insulating perovskite oxide. When electronically doped, $\mathrm{SrTiO}_{3}$ is one of the lowest carrier-density superconductors [2]. Superconductivity arises when mobile electrons form Cooper pairs. In n-doped $\mathrm{SrTiO}_{3}$, these electrons have a so-called $t_{2 \mathrm{~g}}$ orbital character, derived from the titanium $3 d_{x y}$, $3 d_{\mathrm{yz}}$ and $3 d_{\mathrm{xz}}$ orbitals, as shown in Figure $1 \mathrm{~b}$. At room temperature, when $\mathrm{SrTiO}_{3}$ is cubic, the $t_{2 \mathrm{~g}}$ states are energetically degenerate, but symmetry breaking effects can lift the degeneracy. A consequence of this was reported in 1980 by the first observation of multiband superconductivity in doped $\mathrm{SrTiO}_{3}$ [3], which undergoes a cubic-to-tetragonal phase transition when cooled down to cryogenic temperatures.

The interest in $\mathrm{SrTiO}_{3}$ as a hosting material for a richness of electronic phases was amplified by the discovery, in 2004 , that interfaces of this material with selected insulators such as lanthanum aluminate $\left(\mathrm{LaAlO}_{3}\right)$ become conducting (see Figure 1a) [4]. Soon it was shown that these interfaces can also exhibit superconductivity [5]. The confinement of the induced two-dimensional electron system at the interface facilitates a modulation of the carrier density, and with this the electronic/magnetic properties, by electric field gating in backgate and/or topgate configurations $[6,7]$. The symmetry breaking at the interface also gives rise to a significant lifting of the orbital degeneracy, as has been studied in detail especially for the (001)-oriented $\mathrm{LaAlO}_{3} / \mathrm{SrTiO}_{3}$. In that case, the in-plane oriented $d_{x y}$ orbital states have a considerably lower energy than the $d_{x z}$ and $d_{y z}$ states.

In their work, Singh et al. explore gate-dependent superconductivity in the less studied (110)-oriented $\mathrm{LaAlO}_{3} / \mathrm{SrTiO}_{3}$ interface. In this system, one can distinguish the lower energy degenerate $d_{x z}$ and $d_{y z}$ orbitals from the $d_{x y}$ states at higher energy in the confining potential well. For considerable negative backgate voltages, giving rise to interface carrier densities below about $10^{14} \mathrm{~cm}^{-2}$, they observe a single superconducting state with a maximal critical temperature of about $0.2 \mathrm{~K}$. For more positive backgate voltages, a Lifshitz transition is crossed, meaning that the $d_{x y}$ orbital at higher energy starts to become occupied as shown in Figure 1c. The first key finding of Singh et al. is that these additional electrons do not join in the existing superconducting condensate but form a second one [1]. To our knowledge, this tunable switching between a single and a multi-condensate superconductor is a premier result in the field of superconductivity. Remarkably, it does not seem to occur at the (001)-interface, studied previously by some of the same authors, which showed single condensate behaviour under varying band occupancies [8].

A second surprise reported by Singh et al. is that the emergence of the second condensate weakens the overall strength of the superconductivity. Measurements of the superfluid stiffness, i.e. the rigidity of the superconducting state against fluctuations in the phase of its wave function, indicate that these fluctuations can proliferate easier. This softens the superconductivity and reduces the critical temperature [1]. These conclusions are based on microwave-resonance experiments, in which the kinetic inductance of the superconductor is probed. Like for the electrostatic tunability of superconductivity, the low superfluid density of $\mathrm{SrTiO}_{3}$ is the key enabler for such experiments, as the kinetic inductance of a superconductor is inversely proportional to that density. We note here that such microwave-resonance experiments can also be of great interest in the characterization of other low-density, low-dimensional superconductors, including the recently discovered magic-angle twisted bilayer graphene [9]. Moreover, the high kinetic inductance and the sensitivity of these materials to changes in the Cooper pair density hold promise for applications in single-photon detectors: a single photon breaking up a Cooper pair should result in a well-detectable shift of the resonance frequency of the microwave circuit.

Based on the gate dependence of the superconducting properties, Singh et al. conjecture that the multi-condensate superconductivity at these (110)-oriented interfaces has an unconventional $s^{ \pm}$symmetry of the order parameter (see Figure 1d), attributed to a repulsive coupling between the sub-bands involved [1]. With this, the two condensates would be characterized by an opposite phase in their combined superconducting wave function. When thinking about 'smoking gun experiments' to further substantiate the present findings and test for an $s^{ \pm}$symmetry, tunnelling spectroscopy studies quickly come to mind. Such experiments can provide further evidence for the formation of a double condensate by 
displaying two different gaps [3] and can reveal additional details on the superconducting state. A special type of tunnelcontact is a Josephson junction, in which both electrodes are superconducting. Such devices could allow phase-sensitive measurements, confirming the sign changes in the superconducting order parameter associated with the $s^{ \pm}$order parameter symmetry, similar to earlier work on the iron-pnictide superconductors [10].

Altogether, the work by Singh et al. provides interesting and useful pieces to the puzzle of understanding the properties and mechanisms of unconventional superconductivity in oxide-based systems, of which the most paramount challenge remains unravelling the long-standing mystery of high- $T_{\mathrm{c}}$ superconductivity in the cuprate perovskites.

Prof. Hans Hilgenkamp and Dr. Sander Smink are in the Faculty of Science and Technology and MESA+ Insititute for Nanotechnology at the University of Twente,

P.O. Box 217,

7500 AE Enschede, The Netherlands

e-mail: H.Hilgenkamp@utwente.nl

\section{References:}

[1]: Singh, G. et al. (2019). Gap suppression at a Lifshitz transition in a multi-condensate superconductor. Nature Materials, this issue [2]: Schooley, J.F. et al. (1965). Dependence of the superconducting transition temperature on carrier concentration in semiconducting $\mathrm{SrTiO}_{3}$. Physical Review Letters 14, 305-307.

[3]: Binnig, G. et al. (1980). Two-band superconductivity in Nb-doped $\mathrm{SrTiO}_{3}$. Physical Review Letters 45, 1352-1354.

[4]: Ohtomo, A. et al. (2004). A high-mobility electron gas at the $\mathrm{LaAlO}_{3} / \mathrm{SrTiO}_{3}$ heterointerface. Nature 427, 423-426.

[5]: Reyren, N. et al. (2007). Superconducting interfaces between insulating oxides. Science 317, 1196-1199.

[6]: Caviglia, A.D. et al. (2008). Electric field control of the $\mathrm{LaAlO}_{3} / \mathrm{SrTiO}_{3}$ interface ground state. Nature 456, 624-627.

[7]: Smink, A.E.M. et al. (2018). Correlation between superconductivity, band filling, and electron confinement at the $\mathrm{LaAlO}_{3} / \mathrm{SrTiO}_{3}$ interface. Physical Review B 97, 245113.

[8]: Singh, G. et al. (2018). Competition between electron pairing and phase coherence in superconducting interfaces. Nature Communications 9:407.

[9]: Cao, Y. et al. (2018). Unconventional superconductivity in magic-angle graphene superlattices. Nature 556, 43-50.

[10]: C.-T. Chen, et al., (2010). Integer and half-integer flux quantum transitions in a niobium-iron pnictide loop. Nature Physics 6, 260264. 
a

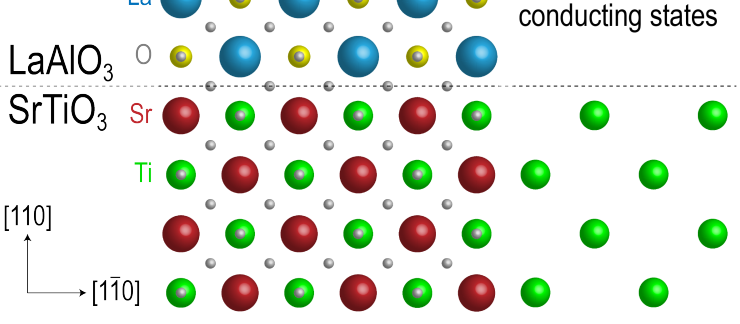

b
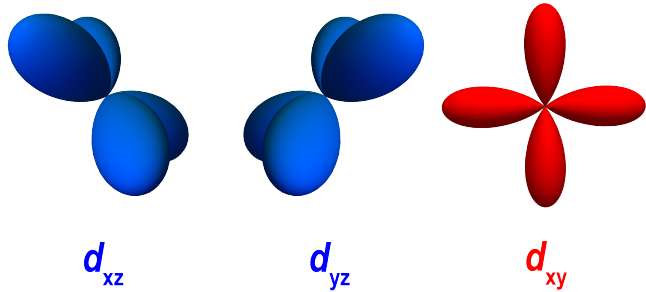

$d_{x z}$

$d_{\mathrm{yz}}$

C

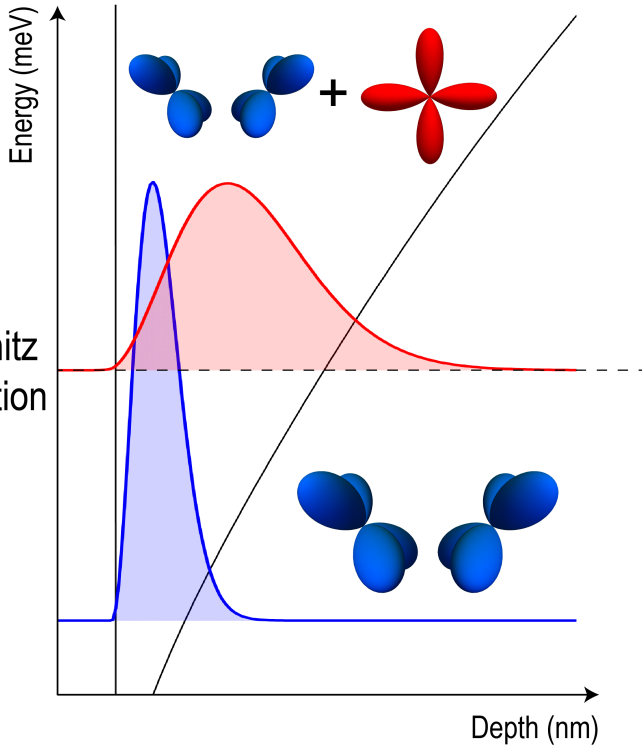

d

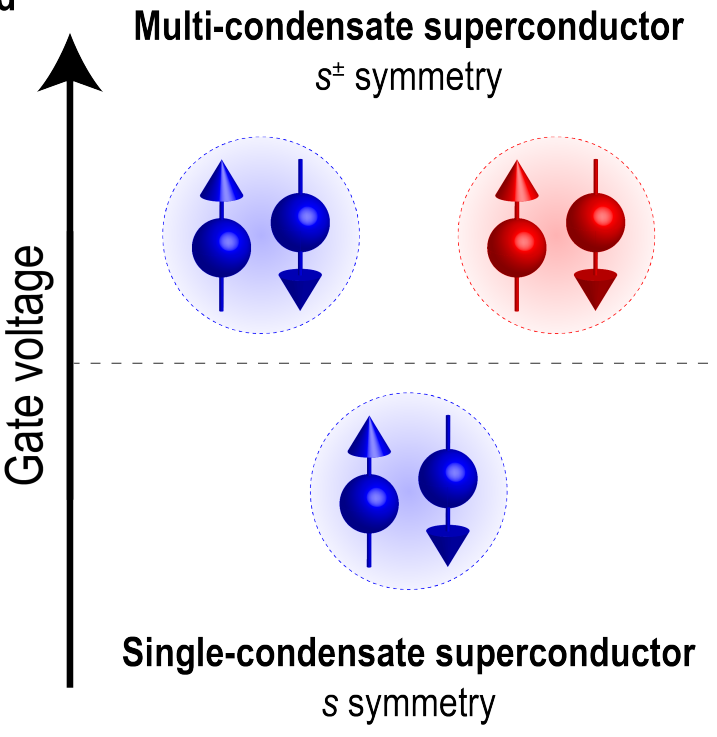

Figure 1 | Electrostatically induced transition between single- and multi-condensate superconductivity at the (110) $\mathrm{LaAlO}_{3} / \mathrm{SrTiO}_{3}$ interface. a Schematic of the (110) $\mathrm{LaAlO}_{3} / \mathrm{SrTiO}_{3}$ interface as investigated by Singh et al. [1], with an indication of the Ti sublattice on which the conducting states reside. Compared to the archetypical (001) interface, the lattice is rotated by $45^{\circ}$. b The $t_{2 g}$ orbitals of the Ti atom, with orientations corresponding to the sublattice in panel (a), which host the (super)conducting states. C Sketch of the quantum well, in which the states are confined to the interface. The confining potential (thin black line) breaks the bulk degeneracy of the $t_{2 g}$ orbitals: by the different orientation with respect to this potential well, the $d_{x y}$ orbital (red) is shifted upwards in energy with respect to the $d_{x z}$ and $d_{y z}$ orbitals (blue). With a gate voltage, charge can be added to the well; the Lifshitz transition denotes a tuning of the Fermi level through the bottom of the $d_{x y}$ band. $\mathbf{d}$ Concurring with the Lifshitz transition, Singh et al. reveal the emergence of a second superconducting condensate, by measuring the kinetic inductance of the interface. From this point onwards, adding more charge to the system weakens the overall superconductivity, as evidenced by a reduction of the critical temperature. 